\title{
Lesion Location: Its Impacts on the Procedural and Postprocedural Outcomes of Unprotected Left Main Coronary Stenting
}

\author{
Woong Kim, $\mathrm{MD}^{1}$, Young-Jo Kim, $\mathrm{MD}^{1}$, Won-Jae Lee, $\mathrm{MD}^{1}$, Sang-Hee Lee, $\mathrm{MD}^{1}$, \\ Geu-Ru Hong, MD ${ }^{1}$, Jong-Seon Park, MD¹, Dong-Gu Shin, MD ${ }^{1}$, Bong-Sup Shim, MD ${ }^{1}$, \\ Yoon-Kyung Cho, $\mathrm{MD}^{2}$, Hyung-Seop Kim, MD², Chang-Wook Nam, MD², Seung-Ho Hur, MD², \\ Yoon-Nyun Kim, MD², Kwon-Bae Kim, MD², Tae-Hyun Yang, $\mathrm{MD}^{3}$, Dae-Kyung Kim, $\mathrm{MD}^{3}$, \\ Sung-Man Kim, MD³, Doo-Il Kim, $\mathrm{MD}^{3}$ and Dong-Soo Kim, $\mathrm{MD}^{3}$ \\ ${ }^{1}$ Cardiology Division, Department of Internal Medicine, College of Medicine, Yeungnam University, Daegu, \\ ${ }^{2}$ Department of Internal Medicine, College of Medicine, Keimyung University, Dongsan Hospital, Daegu, \\ ${ }^{3}$ Department of Internal Medicine, College of Medicne, Inje University, Busan Baik Hospital, Busan, Korea
}

\section{ABSTRACT}

Background and Objectives : Percutaneous coronary intervention (PCI) for unprotected left main coronary artery (LMCA) stenosis is a promising technique in the drug-eluting stent era. However, there is a lack of data as to the impact of the lesion location in the LMCA on the procedural and postprocedural outcomes. The aim of this study is to evaluate the effect of lesion location on the procedural and postprocedural outcomes. Subjects and Methods : From July 2003 to January 2006, we enrolled 82 patients (Males: n=49), who underwent PCI for unprotected LMCA stenosis at Yeungnam University Medical Center, Keimyung University Dong-san Hospital and In-je University Baik Hospital in Busan. The patients were divided into two groups according to the lesion location. Group 1 ( $\mathrm{n}=$ 38) was defined as those patients who had a significant stenosis in the ostium and/or body. Group 2 ( $n=44)$ was defined as those patients had a left main coronary lesion involving a bifurcation. All the patients had a sirolimus eluting stent (Cypher, Cordis) deployed into the LMCA stenosis. Stenting techniques such as the T, crush and kissing stent techniques for treating the LMCA were used at the discretion of the operator and according to the characteristics of the lesion location. The in-hospital outcomes were compared between the two groups and followup coronary angiography was performed after 6 months; the major adverse cardiac events (MACE) and restenosis were analyzed between the two groups. Results: The baseline characteristics between the two groups were not statistically different. The procedural outcomes showed that for the stenting methods, the conventional stent technique was the only one used in all cases of Group 1, but the kissing, T stenting and Crush techniques were also used in Group 2 ( $\mathrm{p}=0.001$ ). The clinical outcomes showed that that there was no statistical difference for the in-hospital MACEs between the two groups, but for the out-of hospital MACEs at 6 month, the target lesion revascularizatin rates, including PCI or bypass graft operation, in Group 1 were higher than those in Group 2 ( $0 \%$ vs $13.6 \%$, respectively $\mathrm{p}=0.043)$. Both groups had one cardiac death each $(2.3 \%$ vs $2.6 \%$ respectively $)$ and there was $1 \mathrm{MACE}$ in Group 1 and 7 MACEs in Group $2(2.6 \%$ vs 16\%, respectively, $\mathrm{p}=0.045)$. The angiographic outcomes at 6 month showed that all 8 significant restenosis cases were included in Group 2 (18\%, 9.7\% in the total population); the restenosis site was the left circumflex artery in all cases. Conclusion : PCI with using drug eluting stents in the left main lesion showed favorable short term outcomes in selected patients. The lesion location is also an important factor for the clinical and angiographical outcomes. (Korean Circulation J 2007;37:419-424)

KEY WORDS : Coronary disease ; Angioplasty ; Treatment outcome.

\section{Introduction}

Coronary artery bypass surgery(CABG) is considered the primary therapy for patients with left main coronary artery(LMCA) disease. The results of several series concerned with percutaneous coronary intervention(PCI)

Received : April 10, 2007

Revision Received : June 5, 2007

Accepted : July 4, 2007

Correspondence : Young-Jo Kim, MD, Cardiology Division, Department of Internal Medicine, Yeungnam University Medical Center,

317-1 Daemyung-dong, Nam-gu, Daegu 705-717, Korea

Tel: 82-53-620-3313,Fax: 82-53-621-3310·E-mail: yjkim@med.yu.ac.kr 
for LMCA disease in the bare metal stent(BMS) era have raised concerns about the safety and mid-term efficacy of PCI because of the relatively high incidence of restenosis and the clinical relevance of recurrent ischemia due to left main stenosis. ${ }^{1-5)}$ The cumulative experience with DES treatment for LMCA has demonstrated an apparent marked reduction in major adverse cardiovascular events(MACEs) as compared with the previous experience after BMS deployment. Accumulating data has repeatedly confirmed that sirolimus eluting stent (SES) implantation has been associated with unparalleled results for reducing the restenosis rates ${ }^{6}$ and neointimal hyperplasia formation. ${ }^{7)}$ Yet little is known about how the lesion location in the LMCA can affect the periprocedural outcomes. The goal of this study was to evaluate the procedural and postprocedural impact of lesion location in the LMCA and the outcomes of undergoing SES implantation for unprotected LMCA stenosis.

\section{Subjects and Methods}

\section{Study population}

From July 2003 to January 2006, eighty two patients (males, $\mathrm{n}=49$ ) who underwent PCI for unprotected left main stenosis at Yeungnam University Medical Center, Keimyung University Dongsan Hospital and Inje Baik Hospital in Busan were enrolled in this study. The LMCA was considered to be unprotected if there were no patent coronary artery bypass grafts in the left anterior descending artery(LAD) or the left circumflex artery(LCX).

\section{Medications and procedures}

All the patients received aspirin $325 \mathrm{mg}$ orally and a loading dose of $300 \mathrm{mg}$ clopidogrel before coronary angiography $(\mathrm{CAG})$ or after PCI in the emergency cases. The patients were routinely treated with aspirin 200 $\mathrm{mg} /$ day, clopidogrel $75 \mathrm{mg}$ /day and/or cilostazol 200 $\mathrm{mg} /$ day. The use of glycoprotein IIb/IIIa inhibitors was left to the discretion of the operator.

CAG was performed after administration of $0.2 \mathrm{mg}$ intracoronary nitroglycerin. Using the guiding catheter for magnification and calibration, quantitative coronary angiography was performed before and immediately after the procedure and at the 6-month follow-up. The stenting techniques for LMCA included the simple "crossover", the "T" stenting, the "crush" stenting and the "Kissing" stent techniques at the discretion of the operator according to the characteristics of the lesion and the anatomy of the left coronary artery. Sirolimus eluting stents(Cypher, Cordis) were used in all the patients. A "kissing" balloon inflation was the final step of the procedure for the bifurcated lesions.

\section{Clinical definitions and follow-up}

Procedural success was defined as residual diameter stenosis $\leq 30 \%$ and the absence of in-hospital MACEs, which were pre-defined as cardiac death, acute myocardial infarction(AMI), target lesion revascularization (TLR) or target vessel revascularization(TVR). Myocardial infarction was diagnosed when the cardiac enzymes (creatinine kinase-MB) were elevated more than threefold times the normal value, with chest pain lasting $\geq 30$ minutes or with the appearance of new electrocardiographic changes. TLR was defined as either surgical or percutaneous reintervention that was done for significant $(>50 \%)$ luminal diameter narrowing within the stent or at the $5 \mathrm{~mm}$ borders proximal and distal to the stent; this was undertaken in the presence of either anginal symptoms or objective evidence of ischemia. TVR was defined as revascularization within the target vessel that encompassed the target lesion. At 6 months after the procedure, all the surviving patients were invited back for angiographic follow-up. Angiographic restenosis was defined as a $\geq 50 \%$ diameter stenosis within the target lesion. The cumulative rates for eventfree survival and MACEs(cardiac death, myocardial infarction and target lesion revascularization) were analyzed during the follow-up period.

\section{Statistical analysis}

The data is expressed as means \pm SD for the continuous variables, and as frequencies for the categorical variables. A two tailed student's test was used to test differences among the continuous variables. The $\chi^{2}$ test was used for comparison of categorical variables. Multivariate Cox analysis was carried out using the baseline clinical and angiographic characteristics to identify the independent predictors of MACEs. The major adverse cardiac event-free survival distributions were estimated according to the Kaplan-Meier method. The log-rank test was used to compare the MACE free survival between the two groups. Probability values $<0.05$ were considered significant. The data was analyzed with using SPSS 12.0 for Windows(SPSS, Chicago, IL., USA).

\section{Results}

\section{Patients and baseline characteristics}

We divided the study population into two groups according to the lesion location: Group $1(n=38)$ was defined as those patients who had a significant stenosis in the ostium and/or body, and group $2(n=44)$ was defined as those patients who had a left main coronary artery lesion involving a bifurcation. The baseline characteristics, including gender, age, diabetes mellitus(DM), hypertension, lipids and the left ventricular function, did not differ between the two groups(Table 1). 
Table 1. Baseline characteristics between the two groups

\begin{tabular}{|c|c|c|c|}
\hline & $\begin{array}{c}\text { Group 1 } \\
(\mathrm{n}=38)\end{array}$ & $\begin{array}{c}\text { Group } 2 \\
(\mathrm{n}=44)\end{array}$ & $\mathrm{p}$ \\
\hline Male & $24(64 \%)$ & $32(19 \%)$ & 0.653 \\
\hline Age (yrs) & $59 \pm 12$ & $61 \pm 9$ & 0.417 \\
\hline DM & $9(24 \%)$ & $8(20 \%)$ & 0.593 \\
\hline Hypertension & 15 (40\%) & $22(50 \%)$ & 0.380 \\
\hline Smoking & $13(34 \%)$ & $12(27 \%)$ & 0.631 \\
\hline LDL-C (mg/dL) & $115 \pm 34$ & $114 \pm 46$ & 0.127 \\
\hline LVEF (\%) & $55 \pm 13$ & $59 \pm 10$ & 0.075 \\
\hline GP IIb/IIIa & 0 & 0 & 1.000 \\
\hline Previous history & & & 0.513 \\
\hline MI & $2(5 \%)$ & $1(2 \%)$ & \\
\hline Intervention & $7(18 \%)$ & $4(9 \%)$ & \\
\hline CABG & $1(3 \%)$ & $1(2 \%)$ & \\
\hline Diagnosis & & & 0.973 \\
\hline Stable angina & 19 (50\%) & $25(57 \%)$ & \\
\hline Unstable angina & $11(29 \%)$ & $12(27 \%)$ & \\
\hline MI & $8(21 \%)$ & $7(16 \%)$ & \\
\hline
\end{tabular}

Table 2. Quantitative coronary angiographic analysis data between the two groups

\begin{tabular}{lccc}
\hline & $\begin{array}{c}\text { Group 1 } \\
(\mathrm{n}=38)\end{array}$ & $\begin{array}{c}\text { Group 2 } \\
(\mathrm{n}=44)\end{array}$ & $\mathrm{p}$ \\
\hline Proximal reference $(\mathrm{mm})$ & $3.6 \pm 0.3$ & $3.6 \pm 0.4$ & $\mathrm{NS}$ \\
Distal reference $(\mathrm{mm})$ & & & \\
$\quad$ LAD & $3.4 \pm 0.3$ & $3.3 \pm 0.3$ & $\mathrm{NS}$ \\
$\quad$ LCX & $2.9 \pm 0.1$ & $2.9 \pm 0.3$ & $\mathrm{NS}$ \\
Average reference (mm) & & & \\
$\quad$ LAD & $3.5 \pm 0.3$ & $3.4 \pm 0.3$ & $\mathrm{NS}$ \\
$\quad$ LCX & $2.9 \pm 0.1$ & $2.9 \pm 0.3$ & $\mathrm{NS}$ \\
Lesion length (mm) & $15.5 \pm 4.4$ & $16.9 \pm 4.8$ & $\mathrm{NS}$ \\
Minimal lumen diameter (mm) & & & \\
$\quad$ Before & $0.7 \pm 0.3$ & $0.7 \pm 0.5$ & $\mathrm{NS}$ \\
$\quad$ After & $3.1 \pm 0.4$ & $2.9 \pm 0.3$ & $\mathrm{NS}$ \\
Diameter stenosis (\%) & & & \\
$\quad$ Before & $80 \pm 10$ & $80 \pm 8$ & NS \\
$\quad$ After & $10 \pm 9$ & $11 \pm 8$ & NS \\
\hline LAD: & &
\end{tabular}

LAD: left anterior descending, LCX: left circumflex

\section{Procedural outcomes}

The quantitative coronary angiographic data is shown in Table 2. Comparison of the angiographic characteristics between the two groups showed that group 2 had a greater number of diseased vessels and lesions with stenosis greater than $50 \%$ (Table 3 ). Table 4 shows the clinical characteristics during PCI. In group 1, the conventional stenting technique was used for all patients, but in group 2, the numbers of kissing stent, T-stent and crushing stent techniques that were used were greater
Table 3. Coronary angiographic findings and differences between the 2 groups

\begin{tabular}{cccc}
\hline & $\begin{array}{c}\text { Group 1 } \\
(\mathrm{n}=38)\end{array}$ & $\begin{array}{c}\text { Group 2 } \\
(\mathrm{n}=44)\end{array}$ & $\mathrm{p}$ \\
\hline Other site lesion & $(>50 \%$ stenosis except & LMT $)$ & 0.001 \\
LAD & $6(16 \%)$ & $12(28 \%)$ & \\
LCX & $4(11 \%)$ & $1(3 \%)$ & \\
RCA & $2(6 \%)$ & 0 &
\end{tabular}

PCI other lesion ( $>50 \%$ stenosis except LMT)

$\begin{array}{llc}\text { LAD } & 4(11 \%) & 6(14 \%) \\ \text { LCX } & 3(8 \%) & 1(3 \%) \\ \text { RCA } & 1(3 \%) & 0\end{array}$

LAD: left anterior descending, LCX: left circumflex, RCA: right coronary artery, PCI: percutaneous coronary intervention, LMT: left main trunk

Table 4. Procedural characteristics compared between the two groups

\begin{tabular}{lccc}
\hline & $\begin{array}{c}\text { Group 1 } \\
(\mathrm{n}=38)\end{array}$ & $\begin{array}{c}\text { Group 2 } \\
(\mathrm{n}=44)\end{array}$ & $\mathrm{p}$ \\
\hline Methods of stenting & & & 0.001 \\
$\quad$ Conventional & $38(100 \%)$ & & \\
Cross-over & & $19(44 \%)$ & \\
Kissing & & $17(39 \%)$ & \\
$\quad$ T-stenting & $3(7 \%)$ & \\
$\quad$ Crushing & & $5(12 \%)$ & \\
Final kissing balloon & $1(3 \%)$ & $31(70 \%)$ & 0.001 \\
IABP & 0 & $1(3 \%)$ & 1.000 \\
\hline IABP: intraaortic balloon pump & &
\end{tabular}

than those of group 1 and final kissing balloon techniques were used more often in group 2 than in group 1 (Table 4).

\section{In-hospital outcomes}

The in-hospital MACEs was compared between two groups and they showed no statistical differences (Table 5).

\section{Out-of hospital outcomes at six month}

The out-of-hospital MACEs and total MACEs were compared between the two groups at 6 month. For the out-of hospital MACEs, the target lesion revascularization rates, including the PCIs or bypass graft operations in group 1, were higher than those in group $2(0 \%$ vs $14 \%$, respectively, $p=0.043$ ), and both groups had one cardiac death each $(2.6 \%$ vs $2.3 \%$, respectively); for the total MACEs, there was 1 in group 1 and 7 in group 2 ( $2.6 \%$ vs $16 \%$, respectively, $p=0.045$, Table 5 , Fig. 1 ).

\section{Mortalities}

There was one death in each group during clinical follow-up. One patient in group 1 was a 70 year old female who experienced sudden death during sleeping at 34 days after discharge. She was on dual antiplatelet therapy with aspirin $100 \mathrm{mg}$ and clopidogrel $75 \mathrm{mg}$ and 
she hadn't skipped her medications. We presumably thought that late stent thrombosis was the cause of death. Another patient in group 2 was a 55 year old man

Table 5. Comparison of in-hospital MACEs, out-of-hospital MACEs and restenosis after 6 month between the two groups

\begin{tabular}{|c|c|c|c|}
\hline & $\begin{array}{c}\text { Group } 1 \\
(n=38)\end{array}$ & $\begin{array}{c}\text { Group } 2 \\
(n=44)\end{array}$ & $\mathrm{p}$ \\
\hline In-hospital MACE & & & 0.537 \\
\hline PCI & 0 & $1(2.3 \%)$ & \\
\hline CABG & 0 & 0 & \\
\hline MI & 0 & $1(2.3 \%)$ & \\
\hline Cardiac death & 0 & $1(2.3 \%)$ & \\
\hline Out-of hospital MACE & & & 0.043 \\
\hline PCI & 0 & $3(6.8 \%)$ & \\
\hline CABG & 0 & $3(6.8 \%)$ & \\
\hline MI & 0 & 0 & \\
\hline Cardiac death & $1(2.6 \%)$ & 0 & \\
\hline Total MACE & $1(2.6 \%)$ & $7(16 \%)$ & 0.045 \\
\hline Restenosis & 0 & $8(18 \%)$ & 0.017 \\
\hline Restenosis site & & & 0.017 \\
\hline LAD & 0 & 0 & \\
\hline LCX & 0 & $8(18 \%)$ & \\
\hline
\end{tabular}

MACE: major adverse cardiac events, MI: myocardial infarction, PCI: percutaneous coronary intervention, CABG: coronary artery bypass graft, LAD: left anterior descending, LCX: left circumflex

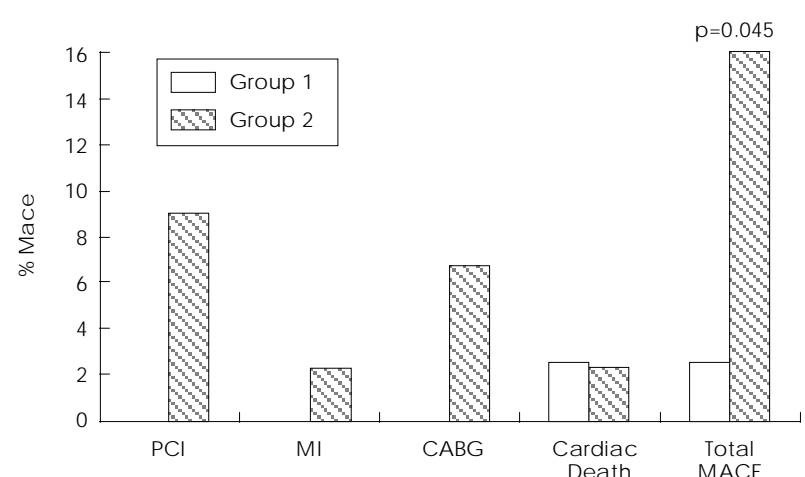

Fig. 1. The cumulative results of the six month angiographic and clinical follow up showed that there were more total major adverse cardiac events (MACEs) in group 2 than that in group 1. MACE: major coronary bypass graft, PCI: percutaneous coronary intervention, AMI: acute myocardial infarction, CABG: coronary artery bypass graft, LAD: left anterior descending, LCX: left circumflex. who developed acute thrombosis and cardiogenic shock after PCI. Aggressive treatment, including an intraaortic balloon pump could not save him. The MACEfree survival at six month is shown in Fig. 2.

\section{Angiographic follow up outcomes at six month}

Six month angiographic follow up was performed in 61 patients $(74.4 \%)$. All the restenosis cases were included in Group 2. The restenosis rate was $18 \%$ for group 2 and $9.7 \%$ for all the patients. The restenosis site was the left circumflex artery in all cases. The clinical and procedural characteristics of the patients with angiographic restenosis are shown in Table 6.

\section{Independent predictors}

The independent predictors of MACEs for unprotected left main coronary stenting, according to univariate regression analysis, were a left ventricular function $<40 \%$, a bifurcated main lesion, DM, a final kissing balloon technique and the methods of stenting. On multivariate regression analysis, DM(OR: 0.068, $\mathrm{p}=$ 0.049 ) and a bifurcated main lesion(OR: 0.012, $\mathrm{p}=$ 0.047) were the independent risk factors of MACEs for LMCA stenting.

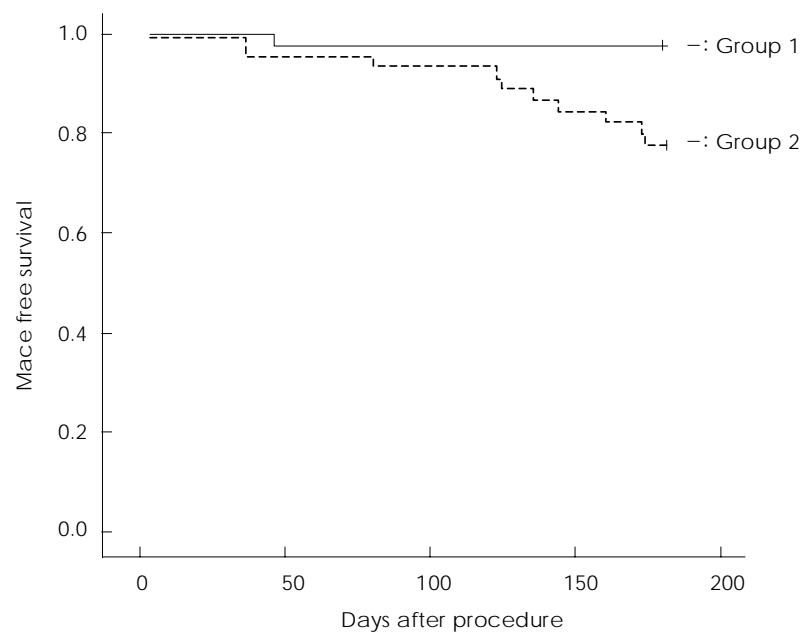

Fig. 2. Major adverse cardiac event (MACE) free survival at six month The results of group 2 were worse than those of group 1. The MACE free survival rate in group 1 was $97 \%$, but that in group 2 was $84 \%$.

Table 6. The eight patients with angiographic restenosis

\begin{tabular}{|c|c|c|c|c|c|}
\hline Age & Gender & Lesion location & Stenting method & Restenosis site & Treatment \\
\hline 58 & $\mathrm{~F}$ & Bifurcation & T-stent & LCX & PCI-Stent \\
\hline 56 & M & Bifurcation & T-stent & LCX & PCI-Stent \\
\hline 57 & M & Bifurcation & T-stent & LCX & PCI-Cutting balloon \\
\hline 42 & $\mathrm{~F}$ & Bifurcation & Crush & LCX & CABG \\
\hline 67 & M & Bifurcation & Kissing & LCX & CABG \\
\hline 67 & M & Bifurcation & Kissing & LCX & CABG \\
\hline 69 & $\mathrm{~F}$ & Bifurcation & Kissing & LCX & Medical therapy \\
\hline 66 & M & Bifurcation & Kissing & LCX & Medical therapy \\
\hline
\end{tabular}

F: female, M: male, LCX: left circumflex artery, PCI: percutaneous coronary intervention, CABG: coronary artery bypass graft 


\section{Discussion}

The prevalence of LMCA stenosis in patients undergoing coronary angiography ranges from $2.5 \%$ to $10 \% .{ }^{8) 9)}$ In contrast, an isolated significant atherosclerotic lesion of the left main coronary artery is rare, with reported incidences of $0.15 \%$ and $0.07 \%$ for all the patients who underwent angiography. ${ }^{10-12)}$ It is widely accepted that coronary artery disease with greater than $50 \%$ left main coronary artery stenosis is a strong indication for early CABG. Many previous studies over the last several years have reported on the safety and feasibility of LMCA stenting. ${ }^{13) 14)}$ Recent evidence is emerging to suggest that PCI with DES may offer a treatment strategy that is both less invasive and has a potentially lower risk for selected patients, and particularly for those patients who are not ideal candidates for CABG because of their comorbidities or advanced age, and also for those patients suffering with acute myocardial infarction(AMI) or cardiogenic shock, and even for the good surgical candidates if there is favorable anatomy for angioplasty. ${ }^{915)}$

DES has emerged as the favored percutaneous treatment modality for LMCA stenosis. Significant reductions in the restenosis rate and the target lesion revascularization(TLR) rate have helped propel DES ahead of bare metal stents(BMSs) for treating subsets of complex lesion. ${ }^{16)}$ Furthermore, improvements in stent deployment techniques with using high balloon pressures, intravascular ultrasound guided stent deployment and glycoprotein IIb/IIIa receptor antagonists have dramatically reduced such complications as subacute stent thrombosis. ${ }^{17-19)}$ A number of registries and nonrandomized studies have demonstrated the effectiveness of DES for treating LMCA stenosis. ${ }^{20-24)}$

LMCA stenosis can be categorized into two major anatomic subsets, depending on whether or not the lesion involves the LMCA distal bifurcation. Several studies have showed that the LMCA distal bifurcation lesion is considered to be unfavorable for percutaneous intervention due to its lower procedural success rate and its higher restenosis rate. ${ }^{25-27)}$

Park et al. ${ }^{20)}$ reported excellent clinical and angiographic outcomes with performing elective sirolimus eluting stent(SES) implantation in a series of 102 patients with unprotected LMCA stenosis, and a distal location of lesion was noted in $71 \%$ of the patients. Six-month angiographic follow-up was completed for $84.3 \%$ of the patients with an overall restenosis rate of $7.0 \%$ and two patients $(2.0 \%)$ required TLR. The oneyear mortality was $0 \%$ and the MACE-free survival was $98 \%$. In another study by Price et al. ${ }^{24)}$ that evaluated the clinical and serial angiographic outcomes of patients undergoing SES implantation for LMCA stenosis, 50 patients were studied via surveillance angiography that was performed at 3 and 9 months' follow up. The group predominantly consisted of distal bifurcation lesions (94\%). TLR occurred in 19 patients (38\%) over a mean follow up of $276 \pm 57$ days. There were two acute stent thromboses and five deaths at 1 year. Angiographic follow up both at 3 and 9 months revealed angiographic restenosis in $23 \%$ of the left main to left anterior descending(LAD) arteries and in 35\% of the left circumflex(LCX) arteries with an overall angiographic restenosis rate of $42 \%$ in any vessel.

We studied 82 patients who underwent SES stent implantation for treating LMCA stenosis. The six month angiographic follow up rate was $74.4 \%$ and the six month clinical follow-up rate was $100 \%$. We divided the study patients into two groups according to lesion location in the LMCA, which was different from the previous studies. From the data acquired from the bifurcation lesion group(Group 2), the restenosis and TLR rates at 6 month were $18 \%(n=8)$ and $13.6 \%$ $(n=6)$, respectively. The six month mortality rate and the MACE rate was $2.3 \%(n=1)$ each in group 1 , and $16 \%$ each $(n=7)$ in group 2 . This showed that when comparing the body and/or ostial lesion in the LMCA, bifurcation lesion has poorer results for restenosis and MACEs, and the body and/or ostial lesion have a more favorable periprocedural results than do the bifurcation lesion. We also found that PCI of the unprotected LMCA with SES is feasible and relatively safe, but this procedure is limited by frequent restenosis that most often involves the left circumflex ostium. This may be due to unique characteristics of the left circumflex ostium as it often contains an acute bend that may predispose to non-apposition of stent struts.

The contribution of final kissing ballon(FKB) to outcomes also cannot be missed in this study. Using a $\mathrm{FKB}$ is associated with more favorable outcomes for bifurcation lesion stenting. ${ }^{28)}$ In our study, FKB was performed in $70 \%$ of the patients with bifurcated lesion, so a FKB was not used in $30 \%$ of the patients. This may have affected the outcomes or the MACEs, but using a FKB was not an independent predictor for LMCA stenting on multivariate analysis. It seems that not only anatomical aspects, but also technical ones may affect the clinical outcomes of LMCA stenting.

In conclusion, PCI using a sirolimus eluting stent in unprotected left main lesion showed favorable short term outcomes in selected patients. The lesion location is also an important factor for the clinical and angiographical outcomes.

\section{REFERENCES}

1) Caracciolo EA, Davis KB, Sopko G, et al. Comparison of surgical and medical group survival in patients with left main coronary artery disease. Circulation 1995;91:2325-34.

2) Takaro T, Peduzzi P, Detre KM, et al. Survival in subgroups of 
patients with left main coronary artery disease. Circulation 1982; 66:14-22.

3) Smith SC, Dove JT, Jacobs AK, et al. ACC/AHA guidelines of percutaneous coronary intervention (revision of 1993 PTCA guidelines) executive summary: a report of the American College of Cardiology/American Heart Association Task Force on practice guidelines. J Am Coll Cardiol 2001;37:2215-39.

4) Silber S, Albertsson P, Marco J, et al. Guidelines for percutaneous coronary intervention. Eur Heart J 2005;26:804-47.

5) Tan WA, Tamai H, Park SJ, et al. Long-term clinical outcomes after unprotected left main trunk percutaneous revascularization in 279 patients. Circulation 2001;104:1609-14.

6) Morice MC, Serruys PW, Sousa JE, et al. A randomized comparison of a sirolimus-eluting stent with a standard stent for $\mathrm{CO}-$ ronary revascularization. $N$ Engl J Med 2002;346:1773-80.

7) Degertekin M, Serruys PW, Foley DP, et al. Persistent inhibition of neointimal hyperplasia after sirolimus-eluting stent implantation: long-term (up to 2 years) clinical, angiographic, and intravascular ultrasound follow-up. Circulation 2002;106:1610-3.

8) Lenzen MJ, Boersma E, Bertrand ME, et al. Management and outcome of patients with established coronary artery disease. Eur Heart J 2005;26:1169-79.

9) Ellis SG, Tamai H, Nobuyoshi M, et al. Contemporary percutaneous treatment of unprotected left main coronary stenoses: initial results from a multicenter registry analysis 1994-1996. Circulation 1997;96:3867-72.

10) DeMots H, Rosch J, McAnulty JH, Rahimtoola SH. Left main coronary artery disease. Cardiovasc Clin 1977;8:201-11.

11) Topaz $\mathrm{O}$, Warner $\mathrm{M}$, Lanter $\mathrm{P}$, et al. Isolated significant left main coronary artery stenosis: angiographic, hemodynamic and clinical findings in 16 patients. Am Heart J 1991;122:1308-14.

12) Miller GA, Honey M, el-Sayed H. Isolated coronary ostial stenosis. Cathet Cardiovasc Diagn 1986;12:30-4.

13) Park SJ, Park SW, Hong MK, et al. Stenting of unprotected left main coronary artery stenosis without anticoagulation: immediate and late outcomes. Korean Circ J 1997;27:947-57.

14) Park JH, Cho YH, Lee SH, et al. Long term clinical follow up in patients with left main coronary disease according to treatment strategies. Korean Circ J 2003;33:568-73.

15) Baim DS, Mauri L, Cutlip DC. Drug-eluting stenting for unprotected left main coronary artery disease: are we ready to replace bypass surgery? J Am Coll Cardiol 2006;47:878-81.

16) Barlis P, Tanigawa J, Kaplan S, di Mario C. Complex coronary interventions: unprotected left main and bifurcation lesions. J Interv Cardiol 2006;19:510-24.

17) Black A, Cortina R, Bossi I, Choussat R, Fajadet J, Marco J.
Unprotected left main coronary artery stenting: correlates of midterm survival and impact of patient selection. J Am Coll Cardiol 2001;37:832-8.

18) Colombo A, Hall P, Nakamura S, et al. Intracoronary stenting without anticoagulation accomplished with intravascular ultrasound guidance. Circulation 1995;91:1676-88.

19) Schomig A, Neumann FJ, Kastrati A, et al. A ramdomized comparison of antiplatelet and anticoagulant therapy after the placement of coronary artery stents. N Engl J Med 1996;334:1084-9.

20) Park SJ, Kim YH, Lee BK, et al. Sirolimus-eluting stent implantation for unprotected left main coronary artery stenosis: comparison with bare metal stent implantation. J Am Coll Cardiol 2005;45:351-6.

21) Chieffo A, Stankovic G, Bonizzoni E, et al. Early and mid-term results of drug-eluting stent implantation in unprotected left main. Circulation 2005;111:791-5.

22) Valgimigli M, van Mieghem CA, Ong AT, et al. Short and longterm clinical outcome after drug-eluting stent implantation for the percutaneous treatment of left main coronary artery disease: insights from the Rapamycin-Eluting and Taxus Stent Evaluated At Rotterdam Cardiology Hospital registries (RESEARCH and T-SEARCH). Circulation 2005;111:1383-9.

23) Lee MS, Kapoor N, Jamal F, et al. Comparison of coronary artery bypass surgery with percutaneous coronary intervention with drug-eluting stents for unprotected left main coronary artery disease. J Am Coll Cardiol 2006;47:864-70.

24) Price MJ, Cristea E, Sawhney N, et al. Serial angiographic follow-up of sirolimus-eluting stents for unprotected left main coronary artery revascularization. J Am Coll Cardiol 2006;47: 871-7.

25) Lee CH, Degertekin M, van Domburg RT, et al. Impact of different anatomical patterns of left main coronary stenting on long-term survival. Am J Cardiol 2003;92:718-20.

26) Wood F, Bazemore E, Schneider JE, Jobe RL, Mann T. Technique of left main stenting is dependent on lesion location and distal branch protection. Catheter Cardiovasc Interv 2005;65: 499-503.

27) Ragosta M, Dee S, Sarembock IJ, Lipson LC, Gimple LW, Powers ER. Prevalence of unfavorable angiographic characteristics for percutaneous intervention in patients with unprotected left main coronary artery disease. Catheter Cardiovasc Interv 2006;68:357-62.

28) Ge L, Airoldi F, Iakovou I, et al. Clinical and angiographic outcome after implantation of drug-eluting stnets in bifurcation lesions with the crush stent technique: importance of final kissing balloon post-dilation. J Am Coll Cardiol 2005;46:613-20. 